\title{
Rapid and Quantitative Determination of S-Adenosyl-L-Methionine in the Fermentation Process by Surface-Enhanced Raman Scattering
}

\author{
Hairui Ren,, ${ }^{1,2}$ Zhaoyang Chen,, ${ }^{1,2}$ Xin Zhang, ${ }^{1,2}$ Yongmei Zhao, ${ }^{3}$ Zheng Wang, \\ Zhenglong $\mathrm{Wu}^{4}{ }^{4}$ and Haijun $\mathrm{Xu}^{1,2}$ \\ ${ }^{1}$ Beijing Bioprocess Key Laboratory, Beijing University of Chemical Technology, Beijing 100029, China \\ ${ }^{2}$ College of Science, Beijing University of Chemical Technology, Beijing 100029, China \\ ${ }^{3}$ Engineering Research Center for Semiconductor Integrated Technology, Institute of Semiconductors, Chinese Academy of Sciences, \\ Beijing 100083, China \\ ${ }^{4}$ Analytical and Testing Center, Beijing Normal University, Beijing 100875, China
}

Correspondence should be addressed to Zheng Wang; zsb@mail.buct.edu.cn and Haijun Xu; hjxu@mail.buct.edu.cn

Received 28 July 2016; Revised 7 September 2016; Accepted 8 September 2016

Academic Editor: Chih-Ching Huang

Copyright (C) 2016 Hairui Ren et al. This is an open access article distributed under the Creative Commons Attribution License, which permits unrestricted use, distribution, and reproduction in any medium, provided the original work is properly cited.

\begin{abstract}
Concentrations of S-Adenosyl-L-Methionine (SAM) in aqueous solution and fermentation liquids were quantitatively determined by surface-enhanced Raman scattering (SERS) and verified by high-pressure liquid chromatography (HPLC). The Ag nanoparticle/silicon nanowire array substrate was fabricated and employed as an active SERS substrate to indirectly measure the SAM concentration. The linear relationship between the integrated intensity of peak centered at $\sim 2920 \mathrm{~cm}^{-1}$ in SERS spectra and the SAM concentration was established, and the limit of detections of SAM concentrations was analyzed to be $\sim 0.1 \mathrm{~g} / \mathrm{L}$. The concentration of SAM in real solution could be predicted by the linear relationship and verified by the HPLC detection method. The relative deviations $(\delta)$ of the predicted SAM concentration are less than $13 \%$ and the correlation coefficient is 0.9998 . Rolling-Circle Filter was utilized to subtract fluorescence background and the optimal results were obtained when the radius of the analyzing circle is $650 \mathrm{~cm}^{-1}$.
\end{abstract}

\section{Introduction}

S-Adenosyl-L-Methionine (SAM) was discovered approximately 60 years ago. Since then, SAM has been identified with a wide spectrum of biological processes ranging from the synthesis of various neurotransmitters in the brain and catecholamine metabolism to gene expression and cell growth, differentiation, and apoptosis, to establish the "SAM empire" called by Cantoni [1-3]. Especially as a biological methyl group donor, SAM is used as a nutritional supplement and as a prescription drug for various diseases, including liver disease [4], osteoarthritis [5], neurologic syndrome [6], and depression [7]. Because SAM could only be ingested from the diet but could not be synthesized endogenously in the human body [4], the demands for SAM in synthetic medication and the nutritional supplements have received wild attention all over the world [8]. Therefore, an effective method for detecting SAM is beneficial to the investigation of the major role of SAM in cell function metabolism and the treatment of clinical disorders. Also the method can be used to optimize the fermentation conditions in fermentation process by monitoring the production of SAM. Recently, Sturgess measured SAM content of the commercially available supplements by high-pressure liquid chromatographyUV diode array detection and showed that the percentage of measured SAM was greater than threefold variation compared to the stated amount on the packaging [9]. Han et al. used high-pressure liquid chromatography (HPLC) to measure the production of SAM and L-isoleucine with $0.67 \mathrm{~g} / \mathrm{L}$ and $13.8 \mathrm{~g} / \mathrm{L}$ in Corynebacterium [10]. Hayakawa et al. reported that the regulation mechanisms are responsible for SAM production by ${ }^{13} \mathrm{C}$-metabolic flux analysis [11]. Notice 
that HPLC, as the major approach for accurate detection of SAM, is rather complex and takes time, implying that it is not suitable for real-time analysis in industry. This stimulates us to seek a novel method which can detect SAM concentration easily and quickly as well as cheaply.

Raman spectroscopy belonging to the molecular vibration spectrum permits the identification of numerous analytes with high discrimination because of its narrow spectral band response. However, the normal Raman signal is extremely weak because of its very small Raman scattering cross-section which limits seriously its applications in fast and sensitive detection. The Raman signals could be efficiently enhanced by surface-enhanced Raman scattering (SERS) technique when molecules were on or near the surface of certain noble metal structures with nanoscale features [12-14]. Silver nanoparticles had drawn particular attention for its potential as SERS substrate due to their highest electrical and thermal conductivities and strong surface plasmon resonance, which make them attractive for use in biochemistry. The enhancement factors (EFs) of SERS can be as much as $10^{3} \sim 10^{7}[15,16]$. Nowadays, SERS, which could minimize photobleaching, peak overlapping, and background signal in complex biological systems, was widely used as a signal-transduction mechanism in biological and chemical sensing [13]. Additionally, biological processes containing abundant materials need a more efficient and sensitive detection method [17]. So the SERS technique was applied to the detection of SAM. In this work, a threedimensional and highly sensitive SERS-active substrate of highly ordered Si nanowire array (Si NWA) decorated by Ag NPs was prepared first, and the SERS spectra of SAM were investigated at various concentrations with the $\mathrm{Ag} / \mathrm{Si}$ substrate (Ag/Si NWA). The limit of detections (LOD) of SAM was found experimentally to be as low as $0.1 \mathrm{~g} / \mathrm{L}$. Furthermore, according to the SERS signals of SAM with a concentration of $0.1 \sim 30 \mathrm{~g} / \mathrm{L}$, the linear relationship between the concentration of SAM and integrated intensity of peak centered at $\sim 2920 \mathrm{~cm}^{-1}\left(S_{2920}\right)$ was established, enabling the qualitative detection of SAM. As the practical application, the fast detection of SAM concentration can be considered as of two steps: $S_{2920}$ was first measured in real solutions and then the SAM concentration was calculated based on the measured $S_{2920}$ and the linear relationship. Meanwhile, the SAM concentrations could also be measured more accurately by HPLC for the verification of the calculation result. The relative deviations $(\delta)$ of the SAM concentration are defined as the ratio of the absolute value of the difference between SAM concentration with the fast detection method and that with HPLC method. The results confirm that $\delta$ are less than $13 \%$.

\section{Materials and Methods}

SAM and the real solutions were obtained from the School of Life Sciences of Beijing University of Chemical Technology (SLS, BUCT). N-type (100) Si wafers $(1 \sim 10 \Omega \cdot \mathrm{cm})$ purchased from the Tianjin Semiconductor Technology Research Institute were used as the substrate material. Acetone, alcohol, HF
( $\geq 40 \%), \mathrm{H}_{2} \mathrm{O}_{2}(30 \%)$, silver nitrate, and deionized water were purchased from the Beijing Chemical Works. All chemical reagents were used as received without further purification.

Before deposition of $\mathrm{Ag}$, Si wafer was first cut into dimensions of $1 \times 3 \mathrm{~cm}^{2}$ and was then ultrasonically cleaned with acetone, ethanol, and distilled water for $5 \mathrm{~min}$, respectively. Next the clean Si wafer was dried in air. The wafer was placed in a mixture of $4.6 \mathrm{M} \mathrm{HF}$ and $0.005 \mathrm{M} \mathrm{AgNO}_{3}$ for $1 \mathrm{~min}$ and then washed with deionized water several times to remove excess Ag particles. Ag nanoparticles (NPs) were then deposited on the surface of the Si wafer by placing the wafer in a mixture $4.6 \mathrm{M} \mathrm{HF}$ and $0.5 \mathrm{M} \mathrm{H}_{2} \mathrm{O}_{2}$ at room temperature for $60 \mathrm{~min}$. After that the Si wafer was taken out steadily. Finally, it was kept in $1 \mathrm{M} \mathrm{AgNO}_{3}$ solution for $1 \mathrm{~min}$ and dried in air.

The concentration of SAM of real fermentation system is from $1 \mathrm{~g} / \mathrm{L}$ to $14 \mathrm{~g} / \mathrm{L}$ [18]. Based on the master facility, the solutions of SAM were prepared with concentration ranges of $0.01 \sim 30 \mathrm{~g} / \mathrm{L}$ by diluting the stock solution of SAM $(30 \mathrm{~g} / \mathrm{L})$ with the minimum concentration which could be tested by SERS. The concentration range is enough to meet the demand for the detection of SAM in real fermentation process. SERS detection of SAM had been completed in 2 hours to ensure the bioactivity. The fermentation liquids were provided by SLS and BUCT with no operation. The cells were disrupted by sonication for $10 \mathrm{~min}$ on ice and then separated by centrifugation at $1000 \mathrm{rpm}$ for $10 \mathrm{~min}$ at $0^{\circ} \mathrm{C}$ from the fermentation liquids. The SAM in supernatant was tested by SERS.

SERS measurements were performed at room temperature on a LabRAM ARAMIS Raman system with the $632.8 \mathrm{~nm}$ laser as excitation. The diameter of the light spot area was $\sim 1 \mu \mathrm{m}$ and the incident power was $9 \mathrm{~mW}$. The spectral resolution was $1 \mathrm{~cm}^{-1}$ and the spectra were recorded with an accumulation time of $15 \mathrm{~s}$. The SERS substrates were preimmersed in corresponding solution for $30 \mathrm{~min}$ to ensure adsorption equilibrium, then rinsed with deionized water, and dried in a vacuum chamber for $5 \mathrm{~min}$ at $25^{\circ} \mathrm{C}$ before each SERS measurement. The range of SERS detection through the whole process was from 550 to $3100 \mathrm{~cm}^{-1}$ at the same condition. The Raman band of a Si wafer at $520.7 \mathrm{~cm}^{-1}$ was used to calibrate the spectrometer.

The background with no chemical information caused by the fluorescence effect in obtained Raman spectrum should be removed. The Rolling-Circle Filter (RCF) is an intuitive filter algorithm to subtract background effect. There are two main advantages of the RCF: (1) it has a single parameter (radius) and (2) there is no restriction on the background shape. This method is based on the geometrical difference between characteristic peaks and background [19-21]. The algorithm and a code written in Matlab language are given in Supplementary Information (see Supplementary Material available online at http://dx.doi.org/10.1155/2016/4910630).

\section{Results and Discussion}

We select highly ordered porous Si NWA as a template to fabricate Ag-based nanostructured SERS-active substrates where large-quantity Ag NPs with appropriate and uniform 


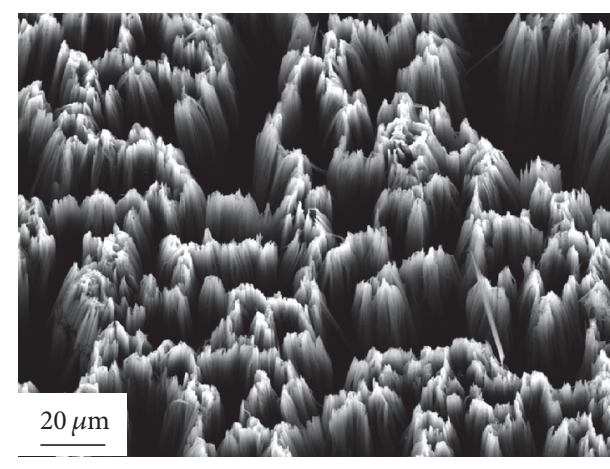

(a)

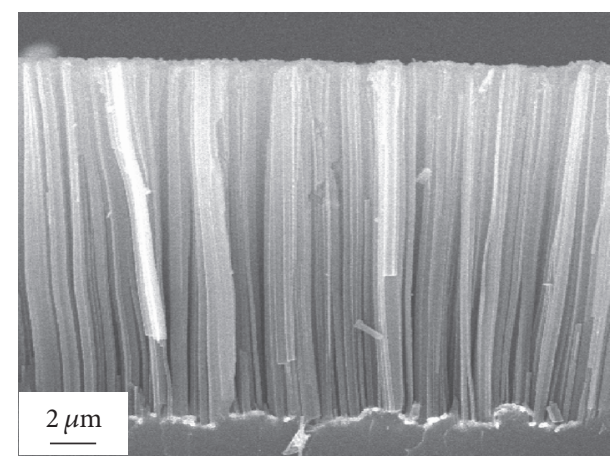

(c)

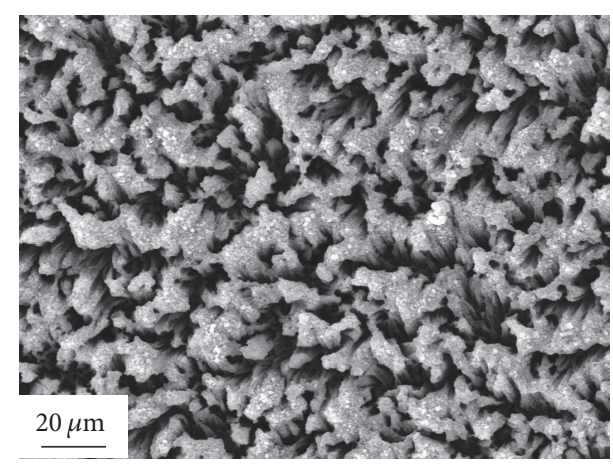

(b)

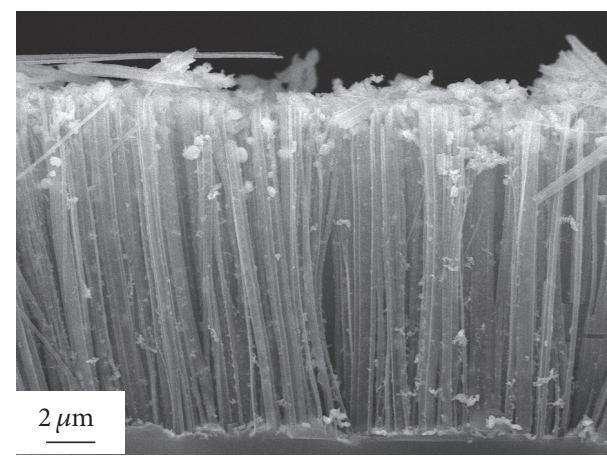

(d)

FIGURE 1: FE-SEM images of a top view and cross-sectional side view of the SiNWA (a), (c); top view and cross-sectional side view of the Ag/SiNWA (b), (d).

size were assembled on the surface of Si NWA via the reaction between Ag ions and $\mathrm{Si}-\mathrm{H}$ bonds. Compared with SERS substrates based on Ag nanoparticle films on monocrystalline Si substrate, the porous Si NWAs have much wider specific areas, allowing an increased loading of Ag NPs and adsorption of target molecules, as well as the higher possibility of forming "hot spots." The typical morphologies of Si NWA and $\mathrm{Ag} / \mathrm{Si} \mathrm{NWA}$ were shown in Figure 1. From the top (Figure 1(a)) and cross-sectional (Figure 1(c)) views of $\mathrm{Si}$ NWA, Si NWs are distributed uniformly on the whole wafers and are oriented perpendicularly to the substrate surface with good uniformity. The diameters of Si NWs are in the range of $300 \sim 1000 \mathrm{~nm}$, while the length is around $10 \mu \mathrm{m}$. From the amplified FE-SEM image of the top view of Si NWA, as shown in the inset of Figure 1(a), it can be observed that the upper ends of Si NWs bend towards each other to form small bunches. The porous nanostructures of $\mathrm{Si}$ NWs provide a much larger surface area, which is of great significance to load Ag NPs and to enhance light trapping. After the Si NWA is decorated, the representative top (Figure $1(\mathrm{~b})$ ) and crosssectional (Figure 1(d)) FE-SEM images of Ag/Si NWA, which also intuitively show that substantive Ag NPs are attached to the surface of each Si NW, and a complex and unique nanocomposite array were formed. The Raman enhancement effect of the Ag/Si NWA substrate is studied, and an EF as large as $3.33 \times 10^{5}$ is calculated, as shown in Supplementary Figure S1. The high EF value indicates Ag/Si NWA has a much

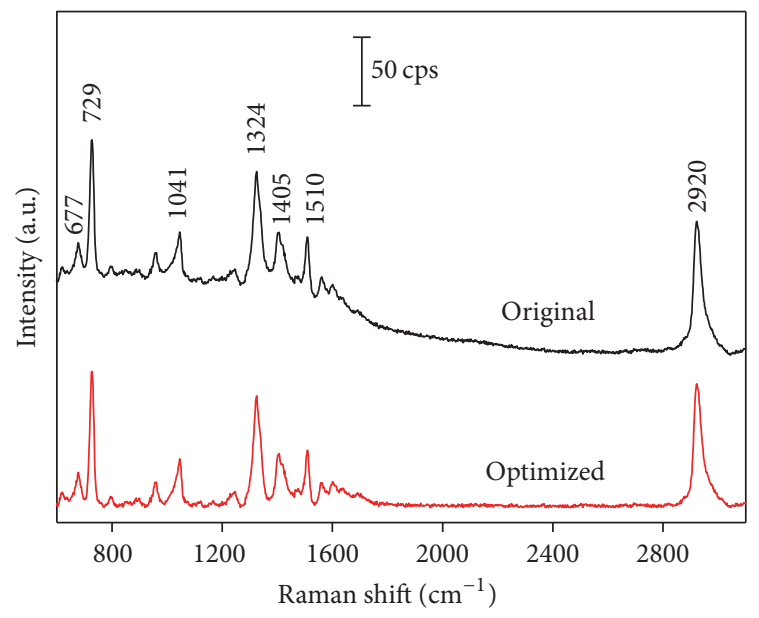

FIgure 2: Model spectrum (black curve: $10 \mathrm{~g} / \mathrm{L}$ ) and the results of applying the RCF method to the curves (red curve: parameters of the analyzing circle $R=650 \mathrm{~cm}^{-1}$ and $R_{x} / R_{y}=16 \mathrm{~cm}^{-1} /$ rel. unit).

better Raman enhancement effect than Si NWA, which is favorable to ultrasensitive detection.

Figure 2 shows the original spectrum (black curve) and the optimized spectrum (red curve) at $10 \mathrm{~g} / \mathrm{L}$ of SAM concentration. It could be found that the fluorescence background with no chemical information was removed by RCF 


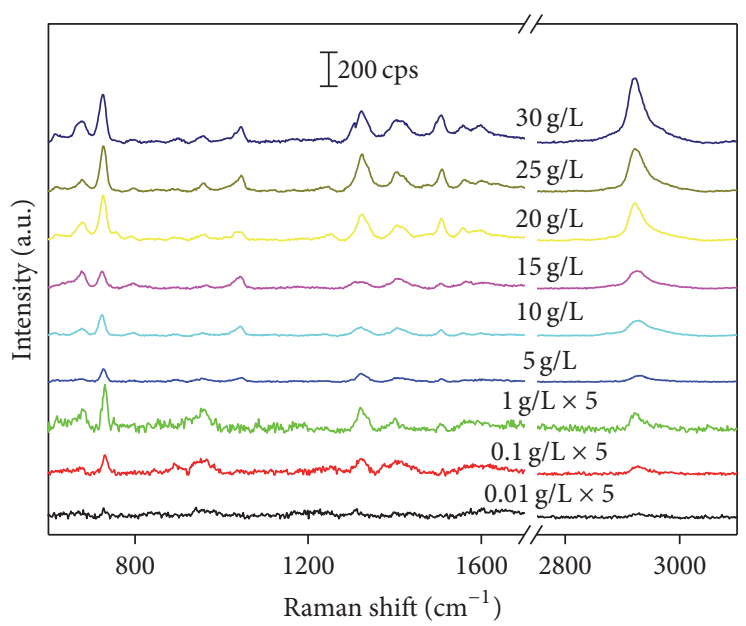

(a)

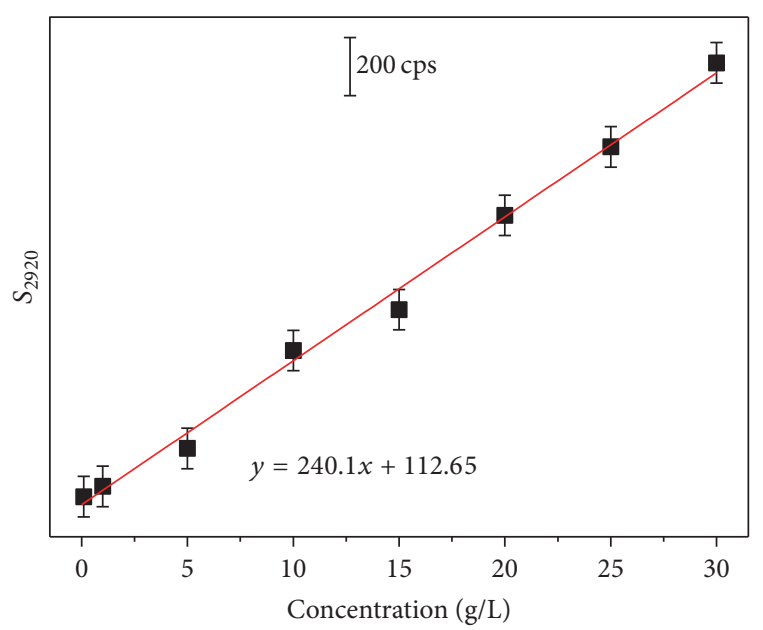

(b)

FIGURE 3: The SERS spectra of SAM solution in various concentrations (a), the quantitative relation curve of the peak intensities centered at $\sim 2920 \mathrm{~cm}^{-1}$ of SAM (b). Each sample was measured for 5 times and the average was calculated.

processing. There is no obvious visible distortion in the optimized curve either. The prominent Raman peaks of SAM at about $677,729,1041,1324,1405,1510$, and $2920 \mathrm{~cm}^{-1}$ were assigned to $v(\mathrm{CS}), v_{s}(\mathrm{CN}), \omega(\mathrm{CH} 2), v(\mathrm{CC}), v_{\mathrm{as}}(\mathrm{CN})$, and $\nu\left(\mathrm{CH}_{3}\right)$, respectively $[22,23]$. The radius of RCF, as a key parameter, was analyzed to achieve the optimal results. Supplementary Figure S2(a) shows the original spectra and the results after applying the RCF with various radii $(50,200$, $400,650,800,1000,1200$, and $1600 \mathrm{~cm}^{-1}$ ). Supplementary Figure S2(b) shows how the difference value between the spectra optimized by the RCF and that with the background subtracted manually varies with the radius of RCF. From Supplementary Figure S2(b), it could be concluded that the optimal radius $R$ is around $650 \mathrm{~cm}^{-1}$. When $R>650 \mathrm{~cm}^{-1}$, the background is partly subtracted, and when $R<650 \mathrm{~cm}^{-1}$, the Raman spectra may have distortion. Therefore, it is verified that $R$ of RCF is significantly greater than the Raman line width and less than the radius of curvature of the background in the spectrum [24].

Usually, the statistical approaches such as partial least square regression (PLSR), Support Vector Machine (SVM) [25], and Gaussian Mixture Discriminant Analysis [26] are combined with principal components analysis (PCA) [27] to quantitatively analyze mixture by Raman or SERS. In the SAM fermentation solution, there is a mass of materials, including the water, inorganic salt, alcohol, ketone with no obvious Raman signals, and SAM with sharp characteristic peaks. With single analyte, we introduce the linear analysis with the relationship between Raman intensity and the concentration of SAM. Figures 3(a) and 3(b) show, respectively, the detection of 0.01 to $30 \mathrm{~g} / \mathrm{L} \mathrm{SAM}$ and the establishment of linear relationship between SAM concentration and $S_{2920}$. As shown in Figure 3(a), the SERS spectra exhibit good regularity, and the intensity recedes with the decrease of SAM concentration. Based on the data presented in Figure 3(a), the relationship curve between the $\mathrm{S}_{2920}$ (average and standard deviation from 8 samples) and the concentration was established. Clearly, the dependence of $\mathrm{S}_{2920}$ on the SAM concentration is nearly linear over the concentration range which was depicted in Figure 3(b). This linear relationship $(y=240.10 x+112.65)$ between $S_{2920}$ and SAM concentration allows for the calibration of our substrate to determine unknown concentration of SAM in solutions. As shown in Supplementary Figure S3, there is no obvious Raman spectrum when the SAM concentration decreases to $0.01 \mathrm{~g} / \mathrm{L}$ whether on $\mathrm{Ag} / \mathrm{Si} \mathrm{NWA}$ or Si NWA substrate, and SERS signals can be merely detected at the concentration of $0.1 \mathrm{~g} / \mathrm{L}$. Thus, LOD of SAM was determined to be $0.1 \mathrm{~g} / \mathrm{L}$, and the linear relationship established above is applicable in the range from $0.1 \mathrm{~g} / \mathrm{L}$ to $30 \mathrm{~g} / \mathrm{L}$, allowing for the quantitative determination of SAM. In order to investigate whether the concentration of SAM could be assessed by SERS technology in real biological samples, we try to study the application of Raman spectrum in real solutions containing SAM at different concentration. The corresponding Raman spectra could be seen in Figure 4. As shown in Figure 4(a), the spectra showed obviously that Raman peaks were centered at $970 \mathrm{~cm}^{-1}$ and $2920 \mathrm{~cm}^{-1}$. The SERS intensity of the mineral salts and glucose contained in real solutions was so weak that it could not be obviously observed. The Raman peaks centered at $970 \mathrm{~cm}^{-1}$ and $2920 \mathrm{~cm}^{-1}$ were assigned to Si NWA and the $\mathrm{CH}_{3}$ symmetric stretching of SAM, respectively. The area of peak centered at $2920 \mathrm{~cm}^{-1}$ showed an increasing trend with concentration of SAM which verified that the Raman peak at $2920 \mathrm{~cm}^{-1}$ was aroused by SAM. Based on the linear relationship in Figure 4(b), the SAM concentration could be calculated and the results were listed in Table 1.

HPLC analysis of real solutions had also been applied to verify the linear function relation and the corresponding results were also listed in Table 1 . The measured value and error of the real solutions were presented, which validates the consistency and the accuracy of the linear relationship. Comparing these data, $\delta$ less than 13\% (including two bigger relative deviations with $12.6 \%$ and $11.13 \%$ and others less 


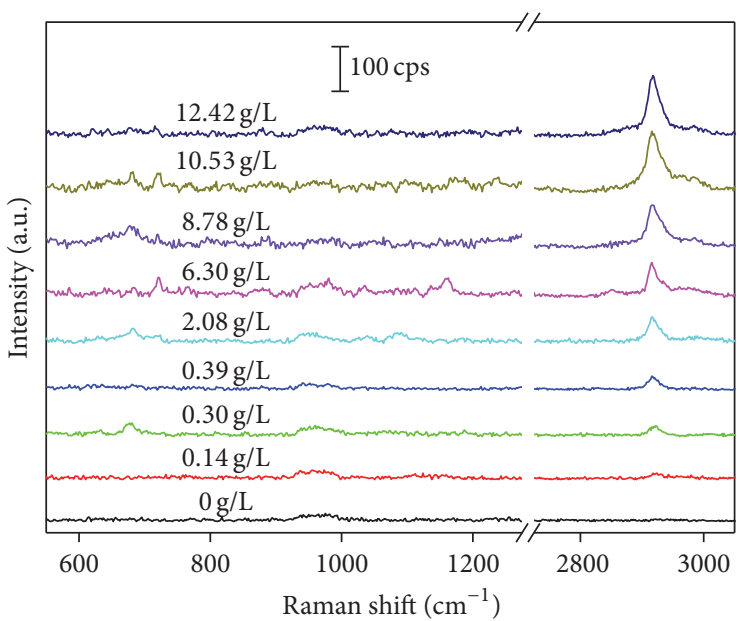

(a)

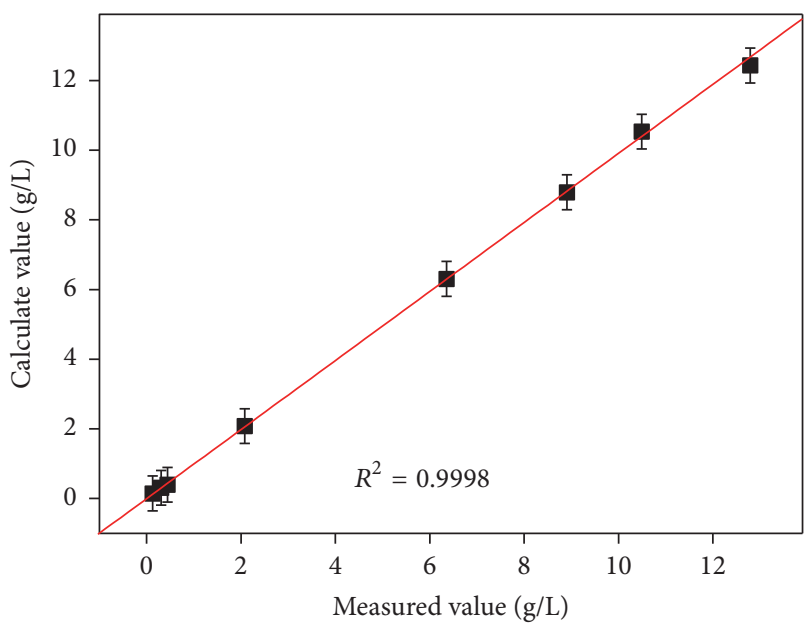

(b)

FIGURE 4: SERS spectra of SAM solution in different concentration (a). Plot for the comparison of the measured value with the calculated value (b). Each sample was measured for 5 times and the average was calculated. The SAM solution of $0 \mathrm{~g} / \mathrm{L}$ was used to prove that the peak centered at $\sim 2920 \mathrm{~cm}^{-1}$ was produced by SAM.

TABLE 1: The calculated and measured results of 8 samples with the relative deviations.

\begin{tabular}{lcccc}
\hline Sample (SAM) & $\mathrm{S}_{2920}$ & $\begin{array}{c}\text { Calculated } \\
\text { values }\end{array}$ & $\begin{array}{c}\text { HPLC } \\
\text { measured } \\
\text { values }\end{array}$ & $\delta(\%)$ \\
\hline 1 & 147.30 & 0.14 & 0.13 & 12.60 \\
2 & 185.76 & 0.30 & 0.31 & 0.29 \\
3 & 206.76 & 0.39 & 0.44 & 11.13 \\
4 & 611.42 & 2.08 & 2.08 & 0.13 \\
5 & 1625.38 & 6.30 & 6.36 & 0.87 \\
6 & 2221.81 & 8.78 & 8.91 & 1.36 \\
7 & 2640.04 & 10.53 & 10.49 & 0.31 \\
8 & 3095.71 & 12.42 & 12.79 & 2.89 \\
\hline
\end{tabular}

than 5\%) were obtained. To present the verification results more intuitively, the predicted concentrations of SAM as a function of the value obtained by HPLC were plotted and shown in Figure 3(b). The correlation coefficient square $\left(R^{2}\right)$ is 0.9998 . Therefore, the determination data were well consistent with the calculated data, indicating that a new method of quantitative determination of concentration of SAM has been developed based on the Ag/Si NWA SERS substrate.

\section{Conclusions}

A new method, surface-enhanced Raman scattering, to detect the SAM concentration rapidly and conveniently was proposed by us. In the investigation carried out here, the linear relationship $(y=240.10 x+112.65)$ between the concentration of SAM and the integrated intensity of peak centered at $\sim 2920 \mathrm{~cm}^{-1}$ was established. Experimentally the LOD of $\mathrm{SAM}$ is $0.1 \mathrm{~g} / \mathrm{L}$. As the concentration of SAM is increased, the integrated intensity of peak increases accordingly. The linear relationship has the same dependence of SAM concentration in aqueous solutions and the real solutions, due to the weak influence on $2920 \mathrm{~cm}^{-1}$ of other contents in biological cells. The relative deviations are less than 13\%, and the correlation coefficient is 0.9998 . This investigation provided a possibility for carrying out rapid detection on SAM. With the mathematic approaches used to establish the predictable relationship including RCF and linear analysis, this research provided a reference to study the relationship between the concentration of SAM and the SERS signals. Furthermore, the results obtained may be applied to the detection for synthesis of SAM and the SAM contention in biological process.

\section{Competing Interests}

The authors declare that there are no competing interests regarding the publication of this paper.

\section{Acknowledgments}

This work was financially supported by the National 973 Basic Research Program of China (2013CB733600), the National Natural Science Foundation of China (21390202, 11104008), the Beijing Natural Science Foundation (4142040), the Beijing Higher Education Young Elite Teacher Project, and Innovation and Promotion Project of Beijing University of Chemical Technology.

\section{References}

[1] D. Mischoulon and M. Fava, "Role of S-adenosyl-L-methionine in the treatment of depression: a review of the evidence," The American Journal of Clinical Nutrition, vol. 76, no. 5, pp. 1158S1161S, 2002. 
[2] J. M. Mato and S. C. Lu, "Role of S-adenosyl-L-methionine in liver health and injury," Hepatology, vol. 45, no. 5, pp. 1306-1312, 2007.

[3] W. A. M. Loenen, "S-adenosylmethionine: jack of all trades and master of everything?" Biochemical Society Transactions, vol. 34, no. 2, pp. 330-333, 2006.

[4] C. S. Lieber, "S-adenosyl-L-methionine: its role in the treatment of liver disorders," The American Journal of Clinical Nutrition, vol. 76, no. 5, pp. 1183S-1187S, 2002.

[5] C. Di Padova, "S-Adenosylmethionine in the treatment of osteoarthritis. Review of the clinical studies," The American Journal of Medicine, vol. 83, no. 5, pp. 60-65, 1987.

[6] T. Bottiglieri, K. Hyland, and K. H. Reynold, "The clinical potential of ademethionine ( $S$-adenosyl-L-methionine) in neurologic disorders," Drugs, vol. 48, pp. 132-157, 1994.

[7] A. L. Williams, C. Girard, D. Jui, A. Sabina, and D. L. Katz, "S-adenosylmethionine (SAMe) as treatment for depression: a systematic review," Clinical and Investigative Medicine, vol. 28, no. 3, pp. 132-139, 2005.

[8] Q. M. Anstee and C. P. Day, "S-adenosylmethionine (SAMe) therapy in liver disease: a review of current evidence and clinical utility," Journal of Hepatology, vol. 57, no. 5, pp. 1097-1109, 2012.

[9] C. P. Sturgess, "Measurement of the S-adenosyl methionine (SAMe) content in a range of commercial veterinary SAMe supplements," Journal of Small Animal Practice, vol. 55, no. 9, pp. 447-450, 2014.

[10] G. Han, X. Hu, and X. Wang, "Co-production of S-adenosyl-Lmethionine and L-isoleucine in Corynebacterium glutamicum," Enzyme and Microbial Technology, vol. 78, pp. 27-33, 2015.

[11] K. Hayakawa, S. Kajihata, F. Matsuda, and H. Shimizu, " ${ }^{13}$ Cmetabolic flux analysis in $S$-adenosyl-1-methionine production by Saccharomyces cerevisiae," Journal of Bioscience and Bioengineering, vol. 120, no. 5, pp. 532-538, 2015.

[12] M. Fan, G. F. S. Andrade, and A. G. Brolo, "A review on the fabrication of substrates for surface enhanced Raman spectroscopy and their applications in analytical chemistry," Analytica Chimica Acta, vol. 693, no. 1-2, pp. 7-25, 2011.

[13] G. Sauer, G. Brehm, S. Schneider et al., "In situ surfaceenhanced Raman spectroscopy of monodisperse silver nanowire arrays," Journal of Applied Physics, vol. 97, no. 2, Article ID 024308, 2005.

[14] Q. Zhou, Y. Yang, J. Ni, Z. Li, and Z. Zhang, "Rapid recognition of isomers of monochlorobiphenyls at trace levels by surfaceenhanced Raman scattering using Ag nanorods as a substrate," Nano Research, vol. 3, no. 6, pp. 423-428, 2010.

[15] B. Sharma, R. R. Frontiera, A.-I. Henry, E. Ringe, and R. P. Van Duyne, "SERS: materials, applications, and the future," Materials Today, vol. 15, no. 1-2, pp. 16-25, 2012.

[16] C. X. Zhang, L. Su, Y. F. Chan et al., “Ag@SiO 2 core-shell nanoparticles on silicon nanowire arrays as ultrasensitive and ultrastable substrates for surface-enhanced Raman scattering," Nanotechnology, vol. 24, no. 33, Article ID 335501, 2013.

[17] A. P. Nash and D. Ye, "Silver coated nickel nanotip arrays for low concentration surface enhanced Raman scattering," Journal of Applied Physics, vol. 118, no. 7, Article ID 073106, 2015.

[18] J. Chu, J. Qian, Y. Zhuang, S. Zhang, and Y. Li, "Progress in the research of $S$-adenosyl-L-methionine production," Applied Microbiology and Biotechnology, vol. 97, no. 1, pp. 41-49, 2013.

[19] N. N. Brandt, O. O. Brovko, A. Y. Chikishev, and O. D. Paraschuk, "Optimization of the rolling-circle filter for Raman background subtraction," Applied Spectroscopy, vol. 60, no. 3, pp. 288-293, 2006.
[20] N. N. Brandt, A. Y. Chikishev, J. Greve, N. I. Koroteev, C. Otto, and I. K. Sakodynskaya, "CARS and Raman spectroscopy of function-related conformational changes of chymotrypsin," Journal of Raman Spectroscopy, vol. 31, no. 8-9, pp. 731-737, 2000.

[21] I. K. Mikhailyuk and A. P. Razzhivin, "Background subtraction in experimental data arrays illustrated by the example of Raman spectra and fluorescent gel electrophoresis patterns," Instruments and Experimental Techniques, vol. 46, no. 6, pp. 765-769, 2003.

[22] M. Tatarkovič, A. Synytsya, L. Št'ovíčková et al., “The minimizing of fluorescence background in Raman optical activity and Raman spectra of human blood plasma," Analytical and Bioanalytical Chemistry, vol. 407, no. 5, pp. 1335-1342, 2015.

[23] B. Rossi, G. Mariotto, E. Ambrosi, and H. L. Monaco, "Raman scattering investigation of selenomethionine replacement in protein SOUL crystals," Journal of Raman Spectroscopy, vol. 40, no. 12, pp. 1844-1848, 2009.

[24] K. I. Zaytsev, I. N. Fokina, A. K. Fedorov, and S. O. Yurchenko, "Sensing of phase transition in medium with terahertz pulsed spectroscopy," Journal of Physics: Conference Series, vol. 486, no. 1, Article ID 012024, 2014.

[25] I. W. Schie, L. Alber, A. L. Gryshuk, and J. W. Chan, "Investigating drug induced changes in single, living lymphocytes based on Raman micro-spectroscopy," Analyst, vol. 139, no. 11, pp. 27262733, 2014.

[26] M. Sattlecker, C. Bessant, J. Smith, and N. Stone, "Investigation of support vector machines and Raman spectroscopy for lymph node diagnostics," Analyst, vol. 135, no. 5, pp. 895-901, 2010.

[27] U. Schmid, P. Rösch, M. Krause, M. Harz, J. Popp, and K. Baumann, "Gaussian mixture discriminant analysis for the single-cell differentiation of bacteria using micro-Raman spectroscopy," Chemometrics and Intelligent Laboratory Systems, vol. 96, no. 2, pp. 159-171, 2009. 

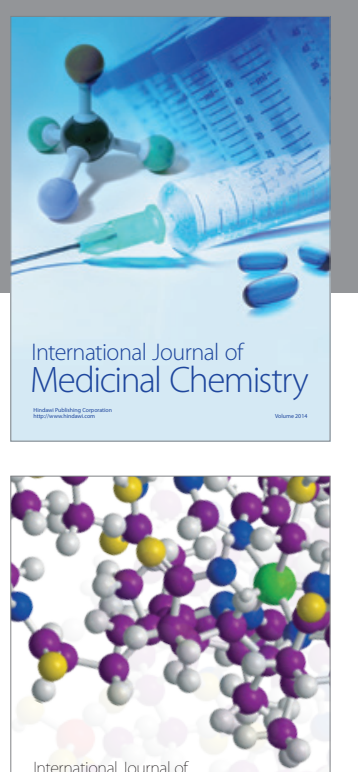

Carbohydrate Chemistry

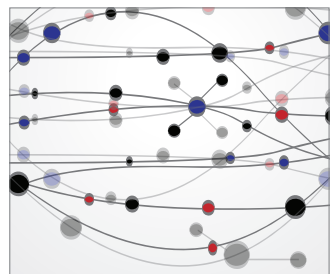

The Scientific World Journal
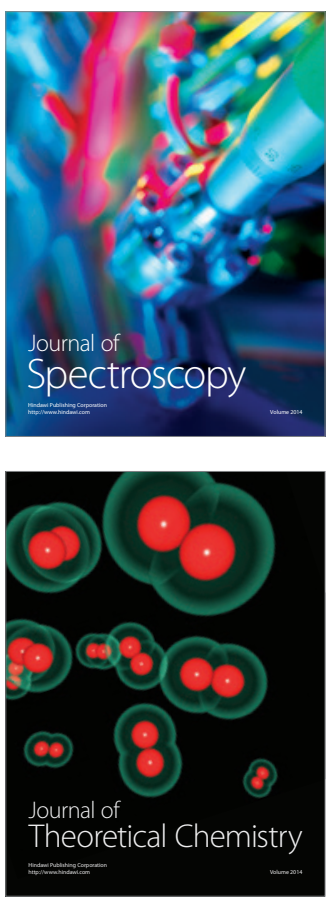
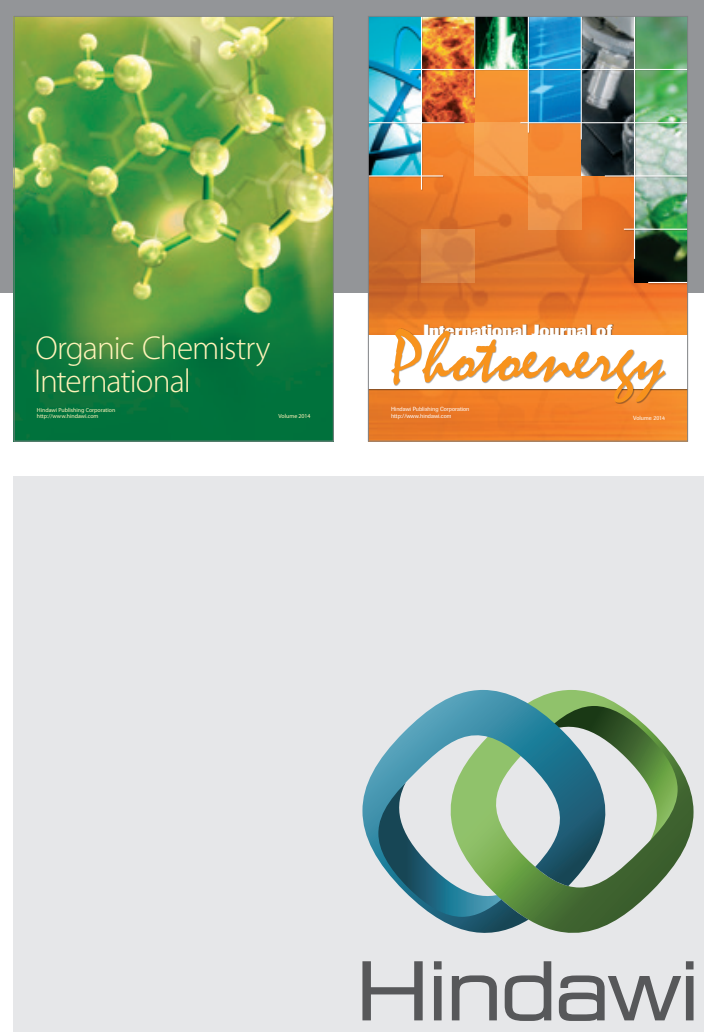

Submit your manuscripts at

http://www.hindawi.com

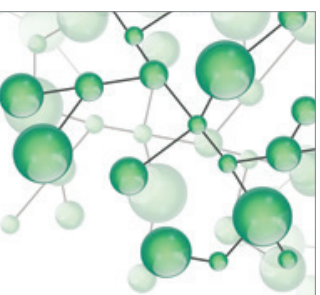

International Journal of

Inorganic Chemistry

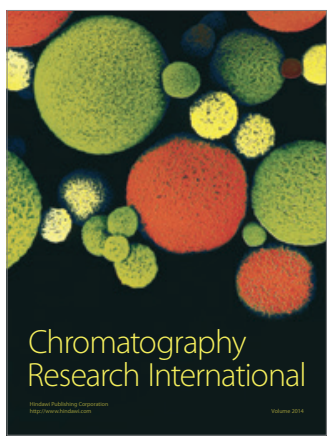

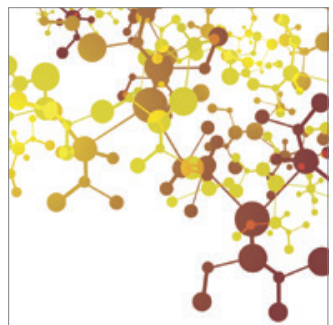

Applied Chemistry
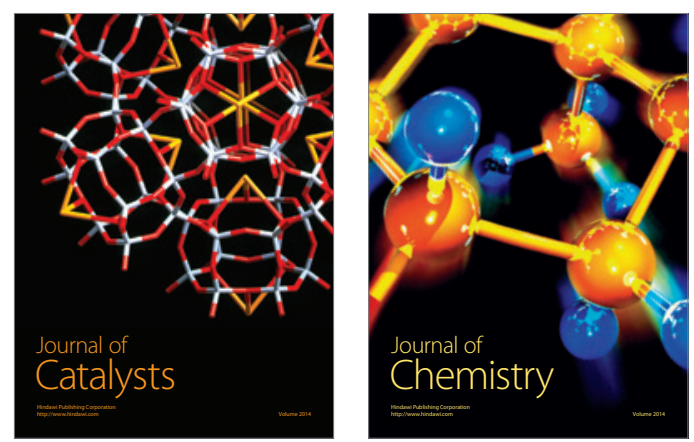
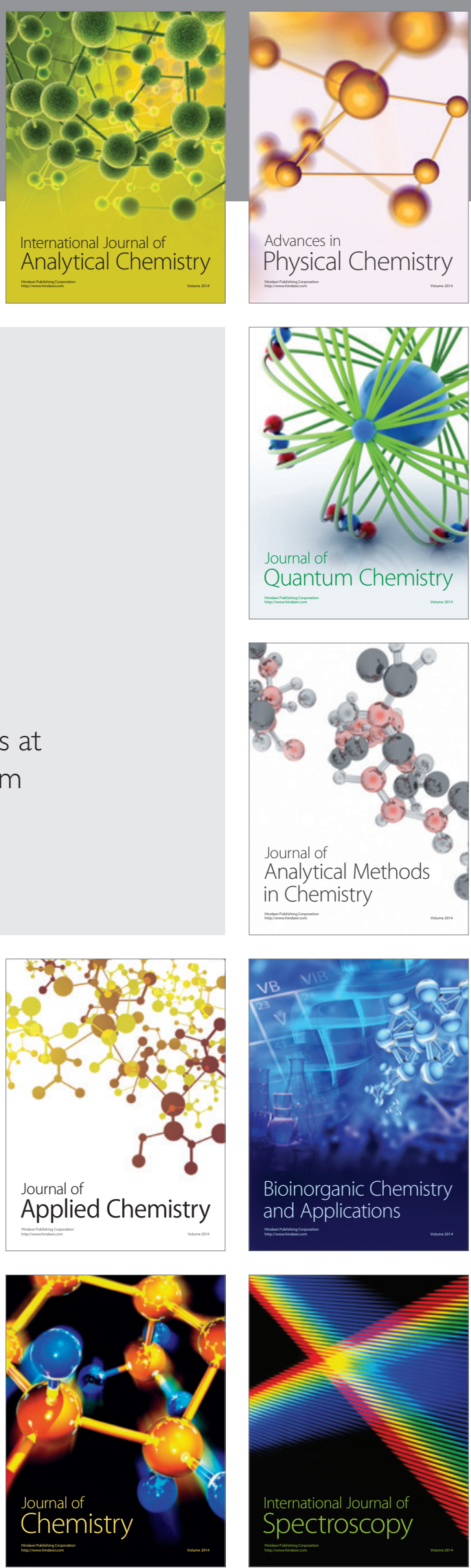